\title{
Book Review: Experimental Human-Computer Interaction - A Practical Guide with Visual Examples
}

\author{
Esra Barut \\ Anadolu University, Turkey
}

\begin{abstract}
Assessment of the interaction that humans form with the system while fulfilling certain computer-based tasks and the improvement of the system resorting to such data are the foundations of human-computer interaction $(\mathrm{HCl})$. To obtain usable data in these studies and to improve the system as a result, design and application processes in the research are of utmost importance. Thus, the book written by Purchase (2015), titled "Experimental Human-Computer Interaction - A Practical Guide with Visual Examples" and published by Cambridge University Press in 2012 in New York was reviewed. The author, who is an experienced $\mathrm{HCl}$ expert, studied several topics such as the visualization of dynamic graphics, multivariate network visualizations, human-centered experiments and application of experimental results in dynamic graphics plotting. The book is based on the course material that the author submitted in 2009. However, during the writing process, the author participated in several seminars and workshops and developed the book through infographics. The author wrote the book to teach operational principles of $\mathrm{HCl}$ experiments using infographics and to define the research area in these experiments. The book has a potential to contribute to researchers that plan a career in research sciences, undergraduate and graduate students and scholars that are interested in $\mathrm{HCl}$.
\end{abstract}

In the first chapter, there is a general definition of the book content. The content includes the tasks the researcher has to fulfill in the process of definition, design, testing and analysis of the research subject in $\mathrm{HCl}$ studies, and reporting the results. This research process is visualized within the framework of iterative system development cycle. The work, true to its objective and content, includes information and steps that would pave the path for researchers that would study in the field of $\mathrm{HCl}$. The author states that the book is focused primarily on experiments in the content. Although the experiments were conducted in the field of $\mathrm{HCl}$, the author shaped the book in a way to address the scientific research process. The author primarily approaches the subject from an analytical perspective; however, the empirical process is explained through a descriptive approach. Generally, the author has a conversational style throughout the book. Thus, individual comprehension of the readers are promoted especially in design and application sections.

The second chapter explains the empirical design process by stressing a rigorous research problem. Furthermore, it is explained that a test set should be prepared to present the research question, conditions, experimental tasks and objects. Thus, it was stipulated that hypotheses on the possible outcomes of the test participants could be created by defining the structure of the experiment. This chapter includes explanations, which could be considered as useful recommendations on how to intellectually plan each step of the experiment and to arrive at findings that could contribute to the field as a result of all empirical research. 
In the third chapter, instructions about the steps that researchers should follow during the experiments are discussed. Thus, it was stated that the participants need to focus on the study process in the experiment, deliberate on what they should do during the experiment and to make decisions accordingly. These decisions concern dividing the participants based on the current conditions, experimental parameters and measures, process material and improvement of the participants. It is also mentioned that, after all decisions are made, pilot experiment should be conducted and ethics committee approval should be obtained. This information would contribute to the researcher in the initial process of the experiment and prevent potential problems that could be experienced during the future processes.

In the fourth chapter, the author discusses the need to collect different type of data and the distribution of data types. The author engages in short descriptions about qualitative and quantitative data and provides examples for data types and how to analyze these types of data. Qualitative and quantitative data are summarized in a table based on data collection and analysis processes and $5 \mathrm{P}$ (performance, preference, perception, process, product) categories. However, data collection process is not detailed. In the chapter, only qualitative analysis methods are discussed.

The fifth chapter addresses the statistical steps that would be beneficial in analysis of quantitative data obtained as a result of comparative experiments. Several quantitative analysis methods, including simple data analysis and comparative and complex analyses that could be conducted on data obtained from $\mathrm{HCl}$ experiments are explained using examples. In the chapter, typical steps of quantitative data analysis are discussed in four phases and identified with an algorithm. It is stated that quantitative measurements do not mean the participants have demonstrated their comprehensive and cognitive activities honestly and these should be supported with qualitative data. Although the expression that the limitations of quantitative data analysis could be compensated with qualitative data guides researchers, mixed method studies are not discussed which could be considered as a limitation of the chapter. The chapter is like a small quantitative data analysis guide. Future editions of the book could include diversified qualitative analysis methods and advanced data analyses that could be utilized in $\mathrm{HCl}$ tests.

In the sixth chapter, how to report the experiment for publication, how to generalize the results and limitations of the study are discussed. In addition, a suitable structure for the reporting process is recommended. Furthermore, views of the critics that shared the same approach with the author are also mentioned. However, mentioning the critics with alternative views could have contributed more to the reporting process. In this chapter, it is interesting to see that an attempt was made to reduce the stereotypes and concerns of the reader through mentioning the necessity to tell a good story in the article following the identification and accurately defining the research question, to discuss the design process and limitations, and to provide information about the validity and reliability of the experiment, which overall was a useful approach.

The seventh chapter provides solutions for probable problems that could be faced in $\mathrm{HCl}$ experiments. It is stated that good ideas should be noted before starting an experiment for risky situations that could occur. These possibilities should carefully be considered, necessary warnings should be issued and all parties should be ready beforehand. The information provided in the chapter specifically includes valuable knowledge for new researchers to protect themselves and take precautions against risks from possible hazards along with possible solutions for the problems they face. 
In the eighth chapter, a modeling is conducted for the relationship between the design and the content of the $\mathrm{HCl}$ experiment, which is finalized with "six golden principles." Furthermore, it is mentioned at the end of the book that there were different methods to test $\mathrm{HCl}$ experiments. It was stressed that every method has advantages and disadvantages. However, to conduct a successful experiment, the method which is most appropriate for the experiment should be selected. The consideration that other researchers would not repeat the mistakes done by the author implies that the book may be contributive to the field.

In short, this book presents the things that should be done in an empirical $\mathrm{HCl}$ study in a sequential way. Besides, ach step is presented in relation to other steps in the process. The book can help scholars interested in empirical $\mathrm{HCl}$ studies in their scientific research. Although the work is quite powerful with respect to the research process, it has limitations in providing theoretical field knowledge. The conversational writing style of the author adds to the comprehensibility of the book. Experiments and discussions are visualized using charts, tables and figures, and therefore, the narrative was materialized to increase retention. The book provides contribution to the literature through filling the gap on how to conduct a research in the field. Further studies in $\mathrm{HCl}$ field may contribute more to the literature through associating the theoretical framework with applied empirical research.

Correspondence: Esra Barut, Doctoral Student, Department of Computer Education and Instructional Technology, Anadolu University, Yunus Emre Campus, Eskisehir, Turkey. 